\title{
Utility of Digital Breast Tomosynthesis with Two-Dimensional Synthesized Mammography Images: A Pictorial Essay
}

\author{
Pradipta C. Hande ${ }^{1}$ Sabita S. Desai ${ }^{2}$ Sarabjeet K. Arneja ${ }^{3} \quad$ Sreedevi Sathian ${ }^{4}$
}

${ }^{1}$ Division of Radiology, Department of Imaging, Breach Candy Hospital Trust, Mumbai, Maharashtra, India

Address for correspondence Pradipta Chakrabarti Hande, MD,

2 Department of Radiology, Breach Candy Hospital Trust, Mumbai, Department of Imaging, Breach Candy Hospital Trust, Bhulabha Desai Road, Mumbai, Maharashtra 400026, India

Maharashtra, India

${ }^{3}$ Department of Surgical Pathology and Cytology, Breach Candy (e-mail: pradipta.hande@gmail.com).

Hospital Trust, Mumbai, Maharashtra, India

${ }^{4}$ Department of Radiodiagnosis, Breach Candy Hospital Trust,

Mumbai, Maharashtra, India

Indian J Radiol Imaging 2021;31:678-688.

\begin{abstract}
Background Mammography has been established as the key modality in the detection and diagnosis of breast cancers. Digital breast tomosynthesis (DBT) has emerged as a mammographic technique which allows improved visualization of abnormalities by reducing the effect of overlapping breast tissue.

Purpose This article is a pictorial essay which highlights the advantages of DBT with two-dimensional (2D) synthesized mammography (2DSM) images, its clinical applications, and its role in breast imaging.

Materials and Methods Selenia Dimensions HD mammography machine performs DBT which acquires a series of low-dose digital mammographic images of the compressed breast followed by full-field digital mammography. Software using

Keywords

- 2D synthesized mammography (2DSM)

- ACR BI-RADS

- digital breast tomosynthesis (DBT)

- FFDM specialized algorithms helps to create a 2DSM image reconstructed from the DBT data set. The images are interpreted on a dedicated work station on high-resolution monitors by the radiologist. American College of Radiology Breast Imaging-Reporting and Data System (BI-RADS) lexicon is used for reporting. High-resolution breast ultrasound which includes evaluation of the axilla is done for all cases.

Conclusion DBT improves detection and better characterization of lesions which thereby increases confidence of interpretation of mammograms and assigning BI-RADS categories for further management.
\end{abstract}

\section{Introduction}

Digital mammography (DM) enables early detection of small nonpalpable, benign, or malignant breast lesions which allows early treatment and thereby has an impact on reduc- ing the mortality of breast cancer. ${ }^{1,2}$ However, the overall sensitivity of DM alone is limited by the superimposed breast tissue that can obscure lesions, resulting in missed cancers especially in dense breasts. ${ }^{3}$ Specificity is reduced by the
DOI https://doi.org/ $10.1055 / \mathrm{s}-0041-1734378$ ISSN $0971-3026$.

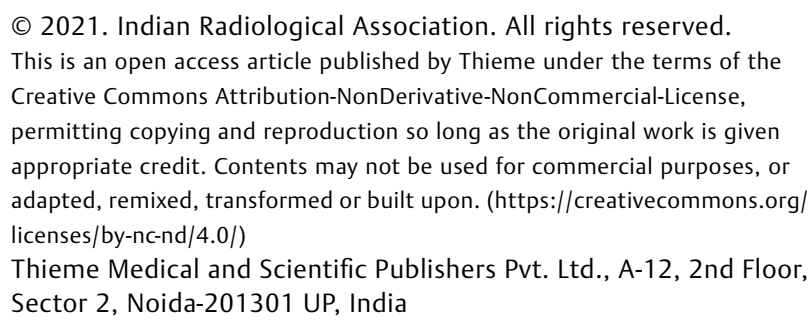


overlapping of fibroglandular tissue, which can give the appearance of lesions that may mimic cancer. ${ }^{2}$ These also lead to unnecessary recalls for further imaging.

Digital breast tomosynthesis (DBT) is a specially modified DM technique that can reduce the masking effect of superimposition of fibroglandular tissue in the breast. ${ }^{2}$ Volumetric data set of projection images is acquired with X-ray tube moving along an arc, which produces multiple tomo-images. The acquired data set can also yield postprocessed reconstructed single two-dimensional synthesized mammography (2DSM) image in each specific view. Besides, routine single exposure full-field digital mammography (FFDM) images can also be done.

When DBT is used in combination mode with FFDM, the patient radiation dose is increased by almost 1.5 to 2 times. ${ }^{4}$

With increasing concerns for patient radiation exposure, studies have shown that DBT with synthesized 2D mammography images gives comparable results with DBT and 2D mammography combination. ${ }^{5,6}$

\section{Materials and Methods}

At our institution, we use the Selenia Dimensions HD mammography system (Hologic Systems, USA, marketed by Trivitron, India), which can perform DBT in combination with FFDM. It has a patented software installed, which can produce 2DSM termed as C-view image, reconstructed from DBT data set, in Tomo HD mode.

Screening and diagnostic mammography using DBT along with FFDM are performed in our department. 2DSM C-view image was evaluated for all cases in each projection for reporting. High-resolution breast ultrasound that includes evaluation of the axilla is done for all cases. Dynamic contrast-enhanced magnetic resonance imaging (MRI) of the breasts was performed in cases where indicated or advised by the surgeon.

\section{Technique and Technical Parameters}

The procedure is similar for routine DM wherein the breast is compressed adequately between the compression paddle and the detector, which remains stationary. Selenia Dimensions HD uses amorphous selenium detectors.

The overhead X-ray tube moves along an arc making an angle called scan angle. As it moves, a series of exposures at different angles are acquired as a digital data set. ${ }^{7}$

Standard mammographic views of craniocaudal (CC) and mediolateral oblique (MLO) are usually done for each breast. At times, mediolateral is obtained at 90 degrees. It is mandatory that adequate compression is given for each breast and no patient motion is ensured. Proper positioning is essential with the nipple seen in profile and inframammary fold in the MLO projection is well visualized. The scan angle in our machine is 15 degrees, with continuous low-dose exposures and scan time of approximately 4 seconds for each standard view.

Combo-mode refers to an acquisition mode in which standard 2D FFDM in neutral perpendicular position is performed after DBT with the tube moving to the center.
2D FFDM and three-dimensional (3D) data sets are perfectly coregistered in the same examination with the same breast compression completed in approximately 10 seconds.

The number of reconstructed slices will depend upon the thickness of the compressed breast and "sections" with desired intervals, which is typically up to $1 \mathrm{~mm}$ thick slices. The images can also be grouped and viewed as a "thick slab." The stack of images is seen with the image receptor with lower numbers being inferior on the $\mathrm{CC}$ view and lateral on the MLO view. The planes of the slices of reconstructed highresolution images are parallel to the detector plate, which supports the breast.

Any object seen in the specific plane of each section in the stack of reconstructed images is in focus, whereas other objects which lie above or below, are out of focus and appear blurred. This helps in better depth localization and allows a "semi-3D" estimation of tissue distribution in $\mathrm{DBT}^{2-7}$

As images are acquired sequentially, tomosynthesis images and 2D FFDM are coregistered which allows lesions to be in the same $x$ - and $y$-axis locations on both sets of images.

Following the exposures, acquired data set is processed by the computer to display tomo-images by filtered back-projection method. The image reconstruction time is 2 to 5 seconds. The reconstructed slices are then transmitted to a diagnostic workstation. High-resolution monitors are recommended for reporting. The radiologist can scroll images slice by slice and localize the abnormal findings with the advantage of visualizing the depth of a lesion in the $z$-axis. At our institution, we use SVDX diagnostic work station with BARCO monitors.

Tomo HD mode is an option available on this machine which allows specialized mathematical algorithms to generate a 2DSM called C-view image from the DBT data set. C-view images are similar to the corresponding FFDM done in the combo-mode, while preserving important details from the tomosynthesis slices.

2DSM makes it possible to reduce compression time from 10 to 4 seconds which eliminates the possibility of motion blurring and is more comfortable for a patient. Without adding the conventional FFDM for every case after performing DBT, it helps in the reduction of overall patient radiation dose as fewer exposures are made..$^{5-7}$

Multiple studies support the ability of DBT with 2DSM alone, with no significant difference in recall rates, cancer detection, and overall diagnostic accuracy compared with DBT performed in combination with 2D FFDM. ${ }^{2,6,8}$

\section{Clinical and Imaging Implications}

American College of Radiology (ACR) Breast Imaging-Reporting and Data System (BI-RADS) lexicon with revised descriptors in the fifth edition helps in the standardization of reports of breast imaging using mammography, ultrasonography, and MRI. ${ }^{9}$

Screening DBT, when combined with 2D DM, has been shown to improve the specificity and sensitivity of a radiology report. ${ }^{10}$ 
DBT images are valuable for identifying masses and distortions better, overcoming the effects of superimposed tissue, which can improve lesion visibility. It allows the radiologist to see the breast in greater detail and identify normal structures, and detect abnormal findings which may be indicative of early breast cancer. It has been found to have decreased the use of additional diagnostic views and reduce recall rates. ${ }^{5,7}$

ACR breast density categories are described as: almost entirely fatty, scattered areas of fibroglandular density, heterogeneously dense, and extremely dense.

The common abnormalities on breast imaging are discussed and interpreted by the radiologist to make a reliable differential diagnosis and for further management of the case as required.

\section{Mass}

DBT images help to characterize the shape, margin, and density of a mass better ( $\mathbf{- F i g . ~ 1 A ) . ~}$. $^{5,8}$

The morphology of lesions is often better defined at DBT indicating them as benign ( - Fig. 2A, B) and malignant, which results in the reclassification of the shape category from the screening examination. ${ }^{10,11}$ Improved visualization allows defining indistinct margins of a lesion that may be obscured by superimposed fibroglandular parenchyma to suggest a suspicious mass, which may change the BI-RADS category (-Fig. 3A, B).

Linear structures and radiating lines are enhanced in 2DSM C-view images which makes the spiculations and architectural distortions (ADs) associated with a malignant mass seen more prominently. ${ }^{5,6}$

The density of a mass is described in comparison to its appearance compared with the expected attenuation of an equal volume of fibroglandular tissue at diagnostic FFDM. ${ }^{7} \mathrm{~A}$ mass that appears to be equal density or high density on 2D mammogram with overlapping tissue layers may appear lower in density on $1 \mathrm{~mm}$ tomo-slice. Fat can be visualized within masses more frequently at DBT, which can lead to possible image misinterpretation. ${ }^{12}$ Fat density in a lesion has a negative predictive value for malignancy in $95 \%$ and in $5 \%$ of cases, malignant lesions may include fat within it. Thin tomosynthesis slices evaluated with the 2DSM image can assess the density of focal lesions better. High-density lesions are an indicator of malignancy. ${ }^{9}$

It is recommended that the most suspicious characteristics in the overall appearance of a mass should determine the requirement of further workup and biopsy rather than one feature alone.

Mammographic BI-RADS 4, 5, or 6 lesions should include lesion size, number of lesions, extent of disease, and presence of contralateral involvement for staging of breast cancer on mammography. ${ }^{13,14}$

DBT can identify additional satellite lesions better along with primary cancer ( - Fig. 4A, B). The presence of multifocal or multicentric disease can impact surgical management and helps in deciding for mastectomy versus breast conservation surgery $(\mathrm{BCS})^{7,13,14}$

\section{Calcifications}

The assessment of type and distribution of calcifications which indicate benign or suspicious categories need to be described carefully for reporting using BI-RADS. ${ }^{9}$

Benign coarse rounded calcifications of liponecrosis are well visualized on DBT with 2DSM and FFDM.

Calcifications with suspicious morphology include amorphous, coarse heterogeneous, fine pleomorphic, or fine

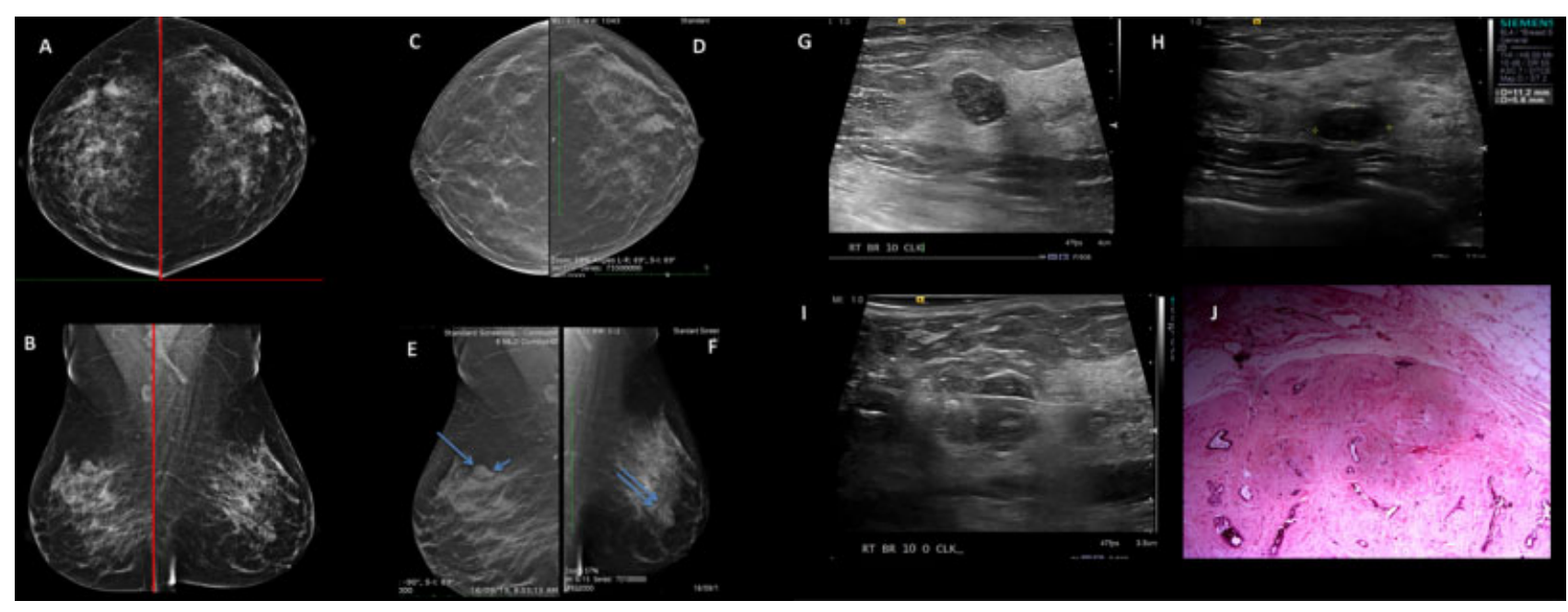

Fig. 1 (A) A 51-year-old lady, postlumpectomy status for benign lesion of right breast for follow-up. (A, B) Full-field digital mammography (FFDM). Craniocaudal (CC) and mediolateral oblique (MLO) views show focal density in superolateral quadrant of right breast better appreciated as oval mass with circumscribed margins (arrow) with microlobulated posterior margin (short arrow) on $1 \mathrm{~mm}$ tomosynthesis slices in (C, D) CC; (E, F) MLO projections Breast Imaging-Reporting and Data System (BI-RADS) 4A. Circumscribed oval mass in the left breast in inferolateral quadrant (double arrows) BI-RADS 2. (G) Ultrasound sonography (USG) of the right breast mass shows oval hypoechoic circumscribed lesion which appears "taller than wide" at 10 o'clock position in right breast. BI-RADS 4. (H) Oval, predominantly anechoic, circumscribed lesion at 4 o'clock position in left breast BI-RADS 2. (I) USG-guided core biopsy done for right breast mass showed fibroadenoma on histopathological examination (HPE). (J) Hematoxylin and eosin (H\&E) 200×. Circumscribed lesion composed of fibroepithelial proliferation. 


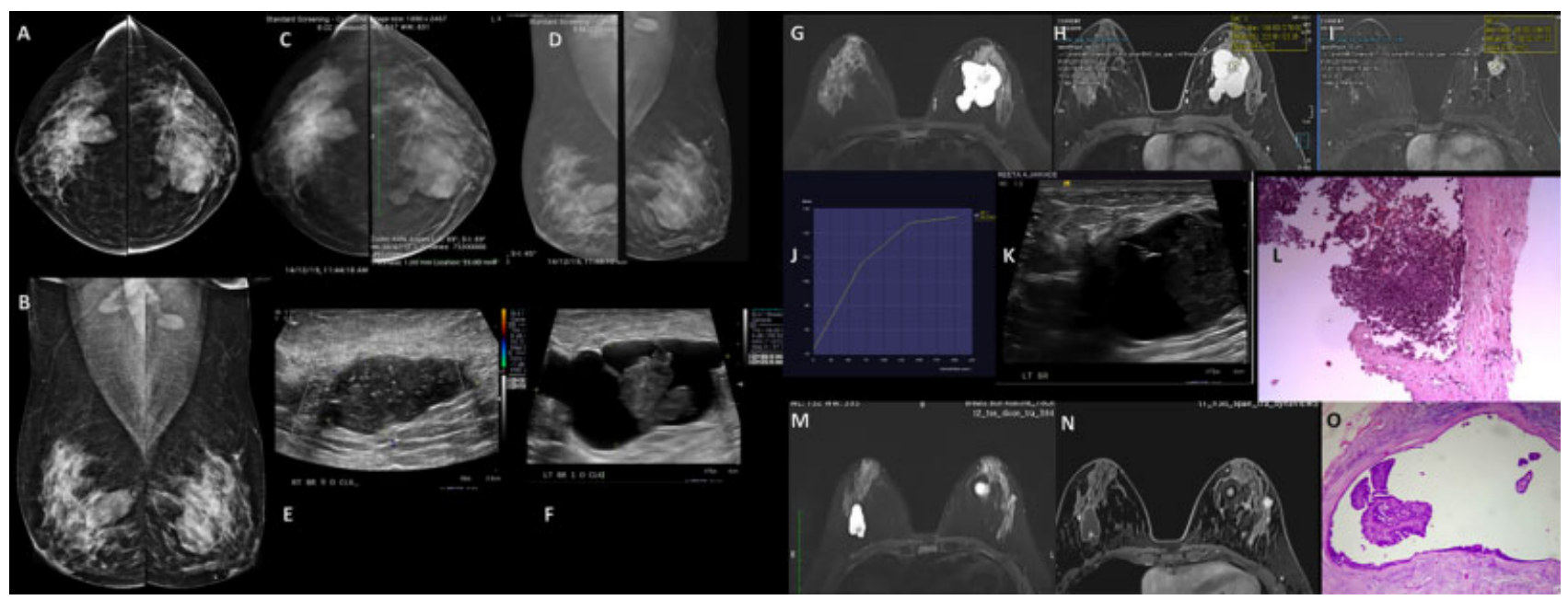

Fig. 2 (A) A 44-year-old lady presented with palpable lump in left breast. Full-field digital mammography (FFDM) (A, B) in craniocaudal (CC) and mediolateral oblique (MLO) views show masses in both breasts. Circumscribed margins (arrows) in both breasts are well appreciated in respective $1 \mathrm{~mm}$ tomosynthesis images with microlobulated margin posteriorly (short arrows in right breast) (C, D). (E) Ultrasound sonography (USG) reveals complex cystic mass at 9 o'clock position in the right breast. (F) Oval lesion with echogenic solid component at 1 o'clock position in the left breast, Breast Imaging-Reporting and Data System (BI-RADS) 4B. (G-I) Contrast-enhanced magnetic resonance imaging (CEMRI) shows circumscribed solid-cystic lesion in the deep retroareolar region of left breast with enhancement of solid component BI-RADS 4. (J) Type 2 kinetics curve. (K) USG-guided core biopsy from solid component of left breast lesion. (L) Hematoxylin and eosin (H\&E) $200 \times$ intraductal (intracystic) papillary carcinoma. (M, N) Predominantly cystic lesion in right breast with tiny solid enhancing component within. (O) Benign intraductal papilloma.

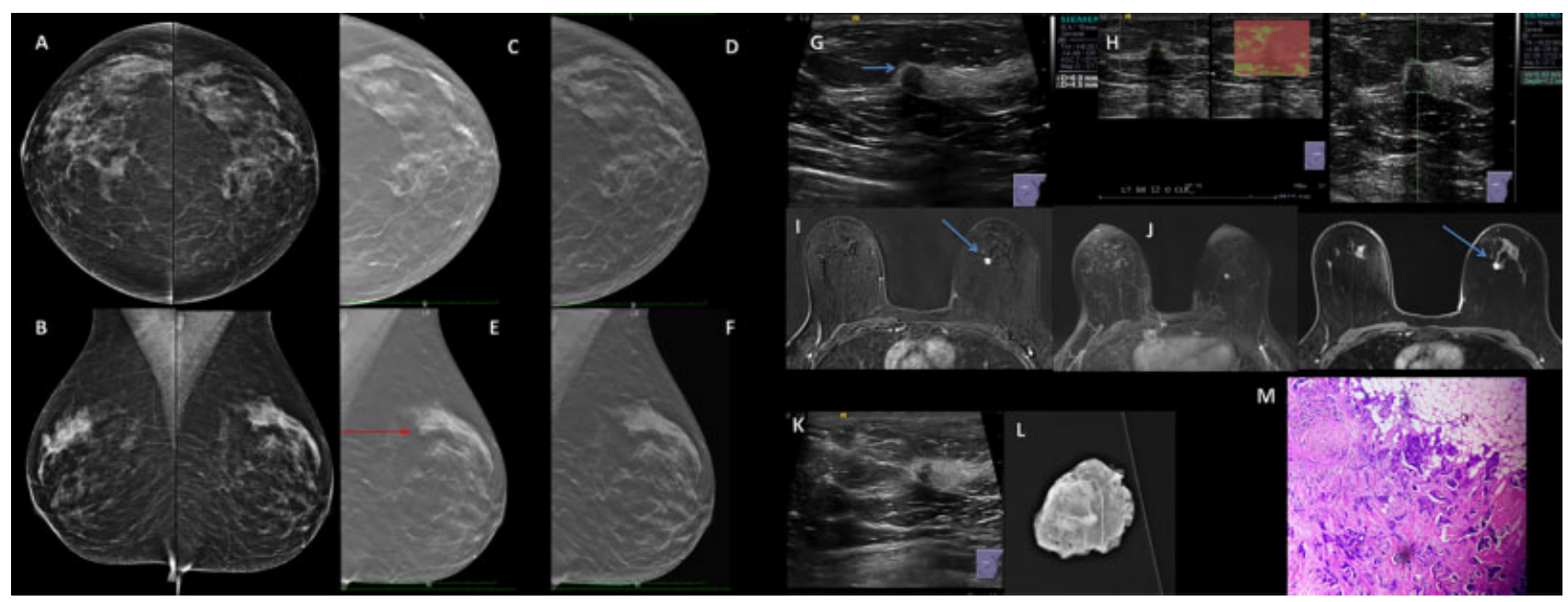

Fig. 3 (A) Screening mammography with family history of breast cancer in mother. Full-field digital mammography (FFDM) of (A, B) craniocaudal (CC) and mediolateral oblique (MLO) views show irregular focal density in superomedial quadrant of left breast which is well appreciated as a mass with spiculated margins in $1 \mathrm{~mm}$ tomosynthesis slices in (C) CC and (E) MLO projections and in (D, F) two-dimensional synthesized mammography (2DSM) images, respectively, Breast Imaging-Reporting and Data System (BI-RADS) 4B. Ultrasound showed (G) hypoechoic lesion (arrow) with microlobulated margin and posterior acoustic shadowing at 11 o'clock position in left breast, hard on elastography (H). Dynamic contrast-enhanced magnetic resonance imaging (DCEMRI) (I, J) shows tiny homogeneously enhancing lesion with irregular margin (double arrows). (K) Preoperative hook wire localization was done. (L) Specimen radiograph confirmed the excision of the lesion. (M) Invasive ductal carcinoma on histopathological examination (HPE).

linear/fine linear branching calcifications. ${ }^{9}$ These have an increased risk for malignancy, with a high probability of up to $50 \%$ in fine pleomorphic, and $78 \%$ in fine linear or fine linear branching calcifications. ${ }^{15,16}$

Diffuse (randomly distributed), regional (occupying more than $2 \mathrm{~cm}$ of breast tissue), grouped (few calcifications in a small area of breast tissue), linear, and segmental appear to be deposited in a duct(s). Grouped calcifications have a minimum of five calcifications within $1 \mathrm{~cm}$ of each other or more within $2 \mathrm{~cm}$ of each other. ${ }^{7,9}$

The visualization of microcalcifications on tomosynthesis images is variable. ${ }^{17-19}$ The limitation is more pronounced for grouped microcalcifications as only a few microcalcifications are in focus in one thin slice. This is a drawback of thin slice tomo-images as suspicious microcalcifications may be missed. 


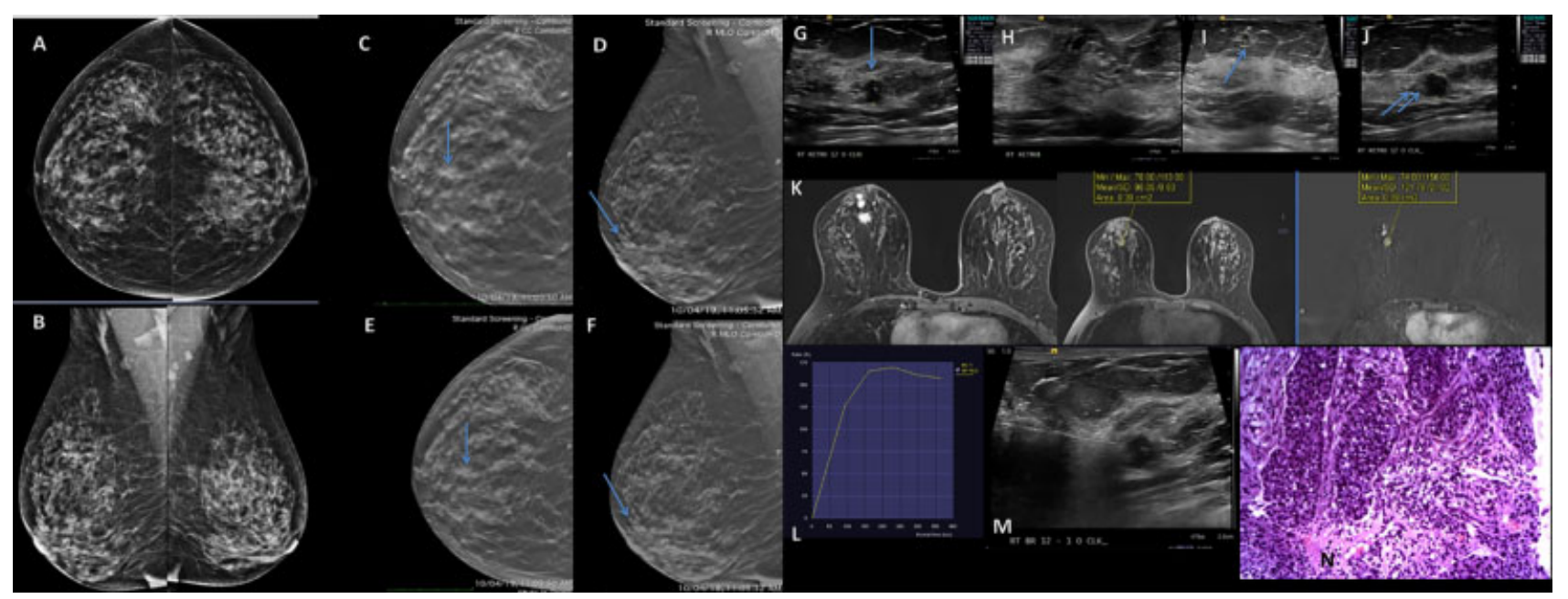

Fig. 4 A 41-year-old lady with retraction of right nipple. (A, B) Bilateral full-field digital mammography (FFDM) of craniocaudal (CC) and mediolateral oblique (MLO) views. Irregular mass in retroareolar region centrally in CC view of right breast. Spiculated margin is better appreciated in $1 \mathrm{~mm}$ digital breast tomosynthesis (DBT) slices (C, D) on CC and MLO projections and in (E, F) two-dimensional synthesized mammography (2DSM) image in the retroareolar region (arrows) with mild skin thickening. (G-J) Hypoechoic mass with irregular margins at 12 o'clock position in the retroareolar region of right breast with associated prominent duct and irregular hypoechoic areas seen at 12 to 1 o'clock position. Dynamic contrast-enhanced magnetic resonance imaging (CEMRI): (K, L) Enhancing spiculated mass in T1W and T2W image in the deep retroareolar region of right breast associated with two smaller enhancing lesions suggestive of satellite nodules anterior to the index mass with type $2 / 3$ kinetic curve; Breast Imaging-Reporting and Data System (BI-RADS) 5. (M) Ultrasound sonography (USG)-guided biopsy. (N) Hematoxylin and eosin (H\&E) $400 \times$ invasive ductal carcinoma.

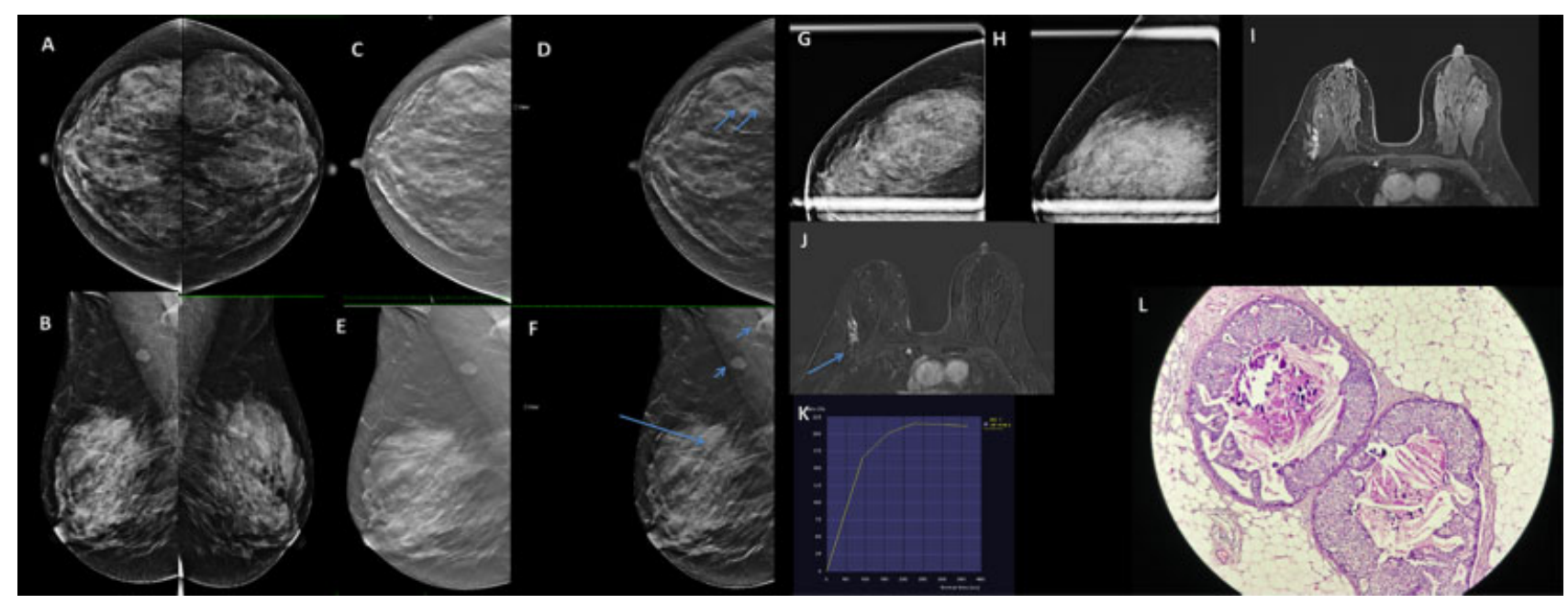

Fig. 5 (A) Screening mammography for a 50-year-old woman. Full-field digital mammography (FFDM) (A, B) of craniocaudal (CC) and mediolateral oblique (MLO) views show bilateral dense breasts with focal asymmetry laterally in right breast well appreciated in $1 \mathrm{~mm}$ tomosynthesis slices in (C, D) CC and MLO. Two-dimensional synthesized mammography (2DSM) images show associated fine pleomorphic microcalcifications in the (E) CC (double arrows) and (F) MLO (long arrow) projections. Breast Imaging-Reporting and Data System (BI-RADS) 4C. Lymph nodes (short arrows) in right axilla. (G, H) Additional spot magnification views show pleomorphic microcalcifications. Dynamic contrastenhanced magnetic resonance imaging (CEMRI) (I, J) T1W axial fat suppressed postcontrast images with subtraction show ill circumscribed nonmass enhancement in the right breast at the 9 o'clock position. (K) Type 2 kinetic curve. Note microcalcifications are not appreciated on MRI. BI-RADS 4. (L) Hematoxylin and eosin (H\&E) ductal carcinoma in situ with microcalcifications.

2D C-view image allows enhanced contrast of "bright points" of calcifications that make microcalcifications more conspicuous (- Fig. 5A, B). This is because the reconstructed image is akin to "thick slab" maximum intensity projection image. ${ }^{2}$ Compared with FFDM, C-view 2D images allowed better visualization of faint fine pleomorphic microcalcifications in our cases (-Fig. 6A, B).

\section{Asymmetry}

Asymmetry refers to an asymmetric appearing tissue in density or appearance seen in a small area of the breast (less than one quadrant).

According to the fifth edition of the BI-RADS atlas, an asymmetry is seen on only one of the two standard mammographic views, either CC or MLO, lacks convex borders, 


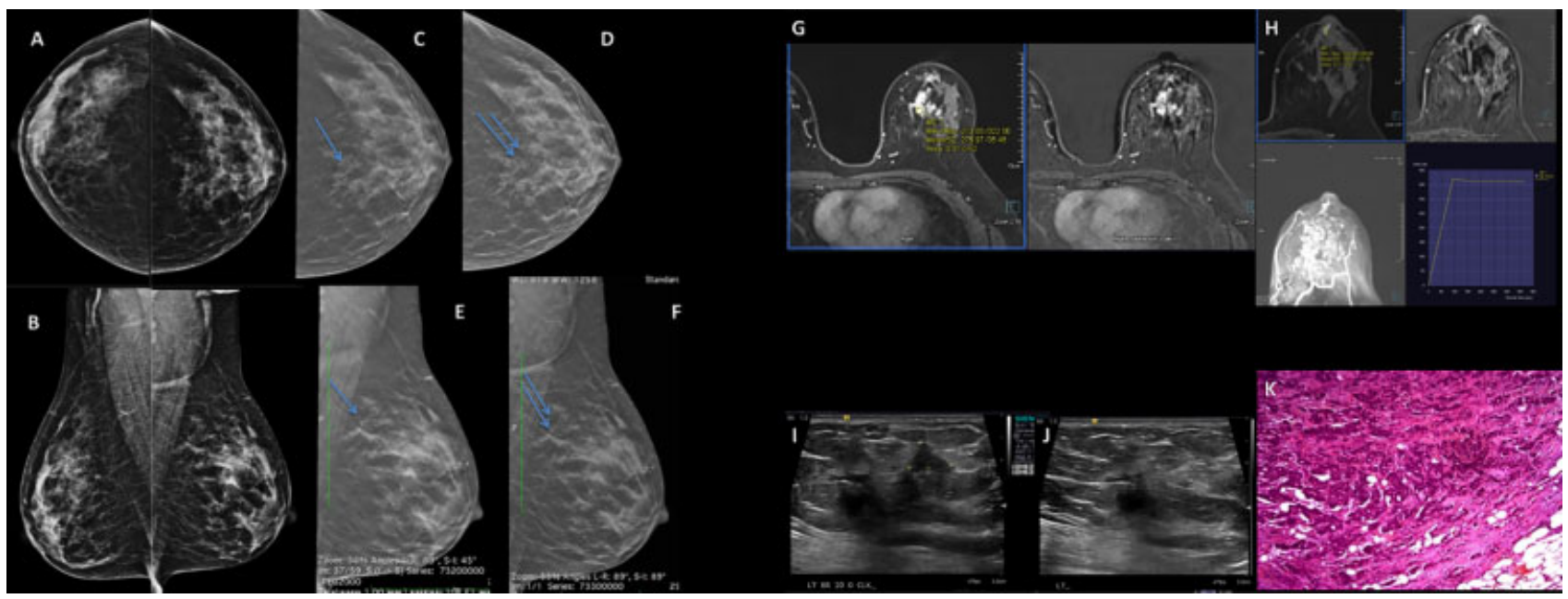

Fig. 6 (A) Screening full-field digital mammography (FFDM) at age 46 years (A, B) in craniocaudal (CC) and mediolateral oblique (MLO) views. Left breast digital breast tomosynthesis (DBT) $1 \mathrm{~mm}$ tomosynthesis slices (C, E) in CC and MLO views show focal asymmetry and architectural distortion (arrows) with fine pleomorphic microcalcifications superiorly toward the posterior aspect of left breast (double arrows) well seen in two-dimensional synthesized mammography (2DSM) (D, F), respectively. Breast Imaging-Reporting and Data System (BI-RADS) 4B. Contrastenhanced magnetic resonance imaging (CEMRI) axial shows $(\mathrm{G}, \mathrm{H})$ linear enhancing duct in left retroareolar region with irregular enhancing nonmass enhancement (NME) involving upper inner and outer quadrants of the left breast which demonstrate type 2 curve on kinetics BI-RADS 5. (I) Ultrasound sonography (USG) shows irregular hypoechoic areas and architectural distortion (AD) with posterior acoustic shadowing involving 11 to 12 o'clock position in left breast. (J) USG-guided core biopsy done. (K) Invasive ductal carcinoma.



Fig. 7 (A) Recent history of left nipple retraction in a 58-year-old lady. Diagnostic full-field digital mammography (FFDM) both breasts (A, B) in craniocaudal (CC) and mediolateral oblique (MLO) views show focal asymmetry in retroareolar region in superomedial quadrant in left breast. (C) $1 \mathrm{~mm}$ tomosynthesis slice reveals small focal densities in the same area in CC projection (long arrow) and in (D) two-dimensional synthesized mammography (2DSM) image (short arrow). (E) However, no obvious mass is seen in additional spot compression view; Breast ImagingReporting and Data System (BI-RADS) 4C. Dynamic contrast-enhanced magnetic resonance imaging (CEMRI). ( $\mathrm{F}-\mathrm{H})$ Axial sections show segmental nonmass enhancement (NME) in superomedial quadrant of the left breast extending from the nipple in the retroareolar region (arrow). (I) Type 2 persistent kinetic curve. (J, K) Segmental NME in coronal and sagittal sections in upper inner quadrant; BI-RADS 5. (L) Histopathological examination (HPE) showed invasive lobular carcinoma.

may or may not contain interspersed fat, and occupies less than one quadrant of the breast. ${ }^{10}$

Global asymmetry is asymmetric breast tissue, as judged relative to the contralateral breast, occupying greater than one quadrant of the breast. ${ }^{10}$

Focal asymmetry has a similar appearance on both the CC and MLO views, lacks convex borders, and may or may not contain interspersed fat. ${ }^{1}$ It is seen on $0.87 \%$ of screening mammograms and has an overall likelihood of malignancy of $0.67 \%{ }^{4}$
DBT can confirm true asymmetry from artifacts as it reduces the effects of tissue overlap and helps to indicate a suspicious lesion (-Fig. 7A, B). Breast cancer seen on tomosynthesis slices were characterized as an asymmetry or focal asymmetry in $20 \%$ cases on FFDM. ${ }^{20}$

The term developing asymmetry has been added to the BIRADS fifth edition and is a focal asymmetry that was not present on the prior mammogram or has increased in size or conspicuity ( $\mathbf{- F i g . ~ 8 A , ~ B ) . ~}{ }^{10}$ The overall malignancy rate of 


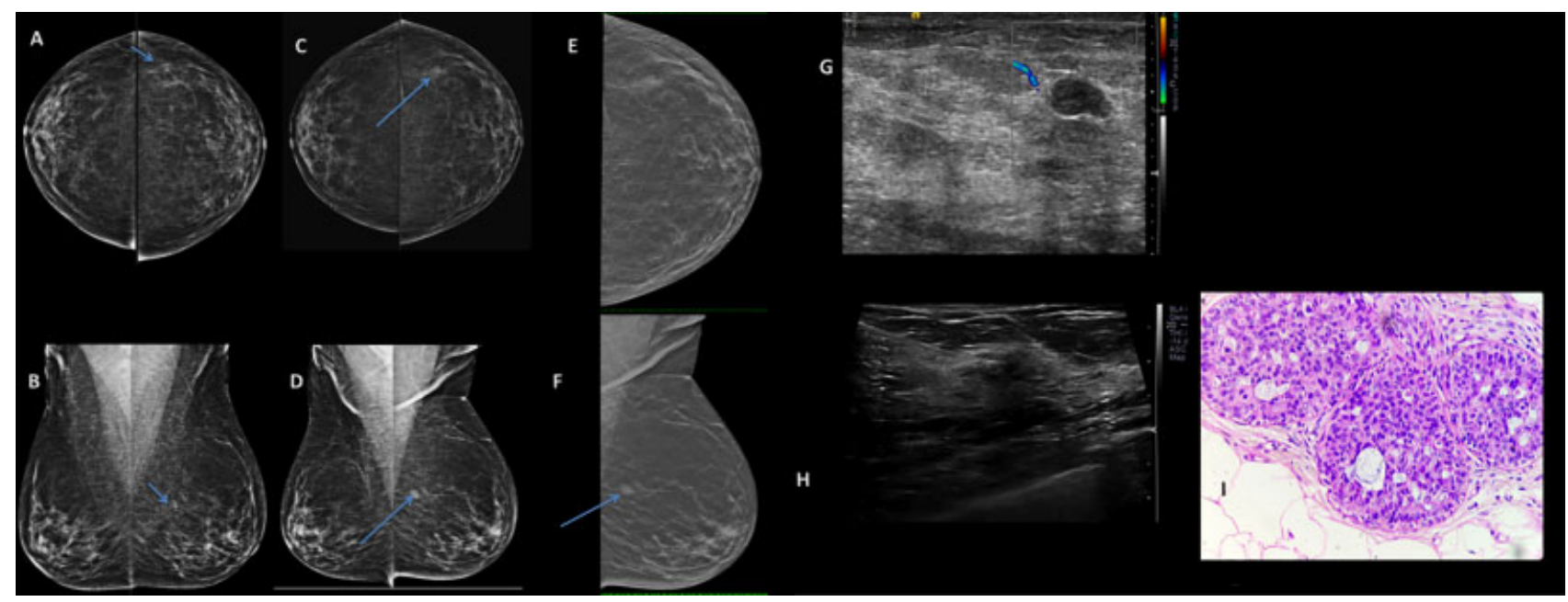

Fig. 8 (A) A 59-year-old lady developing asymmetry (short and long arrows) noted in superolaterally in left breast in consecutive mammograms done after 2 years. Full-field digital mammography (FFDM) in (A, C) craniocaudal (CC) and (B, D) mediolateral oblique (MLO) view. This is seen as a focal density in the superolateral quadrant of the left breast, identified well on respective (E, F) two-dimensional synthesized mammography (2DSM) images. Breast ImagingReporting and Data System (BI-RADS) 4C. (G) Ultrasound sonography (USG) showed a hypoechoic oval mass with microlobulated margin at 2 o'clock position in the left breast; BI-RADS 4. (H) USG-guided biopsy of the lesion was done. (I) Ductal carcinoma in situ.

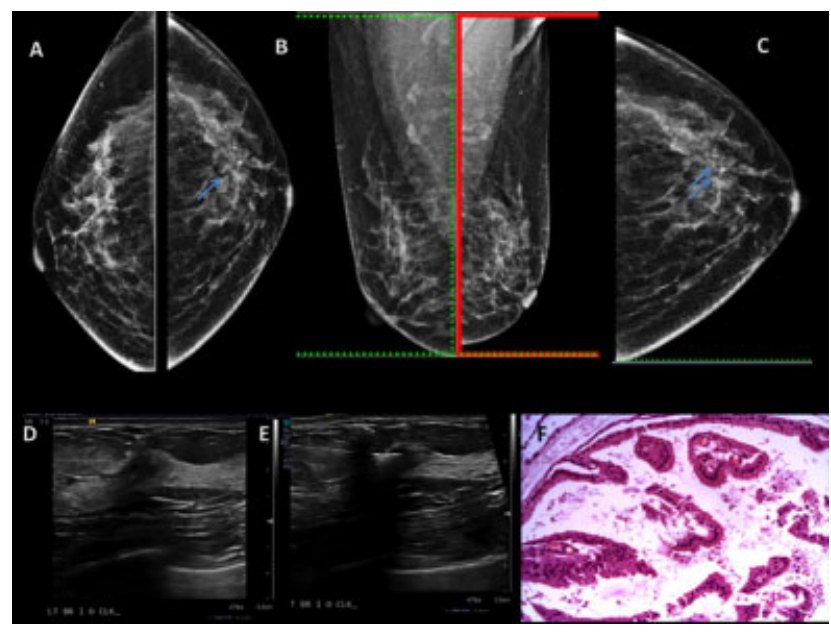

Fig. 9 A 40-year-old lady screening full-field digital mammography (FFDM) of (A, B) craniocaudal (CC) and mediolateral oblique (MLO) view show architectural distortion (AD) (arrow) in the superolateral quadrant of left breast, which is well visualized in two-dimensional synthesized mammography (2DSM) CC image (C) (double arrows) in Cview image. (D) Ultrasound sonography (USG) shows hypoechoic area of architectural distortion with posterior acoustic shadowing; Breast Imaging-Reporting and Data System (BI-RADS) 4B. (E) USG-guided biopsy of the lesion. (F) Hematoxylin and eosin (H\&E) $200 \times$ cystic and papillary apocrine metaplasia.

developing asymmetries in screening and diagnostic populations was $15.4 \%$ in a study by Chesebro et al. ${ }^{20} \mathrm{~A}$ true developing asymmetry should be considered suspicious for malignancy and Price et al recommended further workup. ${ }^{21}$

Additional spot compression views with tomosynthesis can also be performed for specific areas as required to show that true lesions maintain their dimensions because of the desmoplastic response of surrounding tissue in a carcinoma. ${ }^{20}$ These findings are seen better on 2DSM as linear densities radiating from a mass are enhanced. Circumscribed

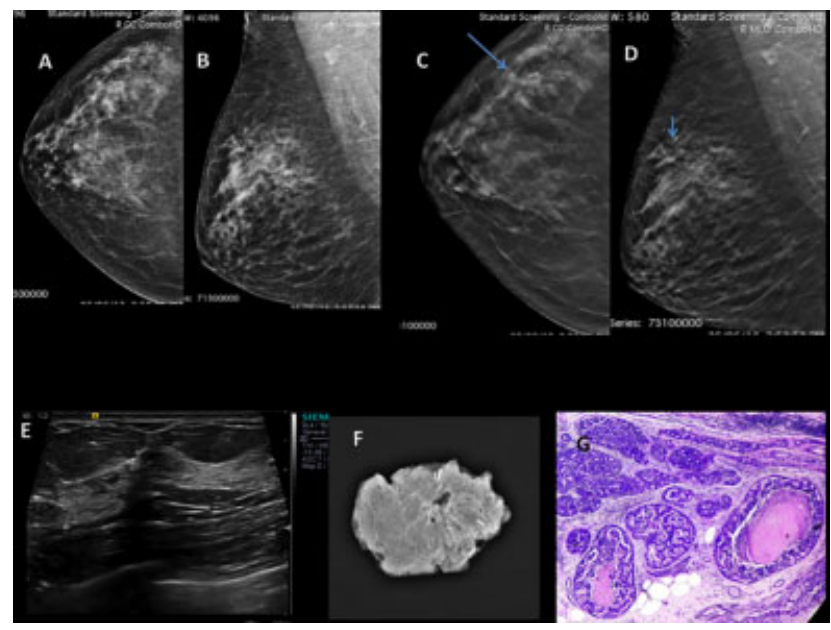

Fig. 10 A 70-year-old lady screening full-field digital mammography (FFDM) of right breast (A, B) craniocaudal (CC) and mediolateral oblique (MLO) views show focal asymmetry in superolateral quadrant. $1 \mathrm{~mm}$ digital breast tomosynthesis (DBT) slice reveals (C) architectural distortion in CC (arrow) with fine pleomorphic microcalcifications (short arrow) appreciated better in MLO projection (D); Breast Imaging-Reporting and Data System (BI-RADS) 4B. (E) Ultrasound sonography (USG) showed architectural distortion (AD) with irregular hypoechoic lesion with posterior acoustic shadowing at 10 'clock position in right breast. (F) Specimen radiograph on excision. (G) Histopathological examination (HPE) showed ductal carcinoma in situ (DCIS).

margins do not, however, imply a mass as benign and individual imaging features cannot be relied upon. ${ }^{7}$

\section{Architectural Distortions}

Abnormality or distortion of normal breast architecture without the identification of a discrete mass, includes spiculations radiating from a point, focal retraction, and altered pattern of the glandular tissue. ${ }^{22}$ 


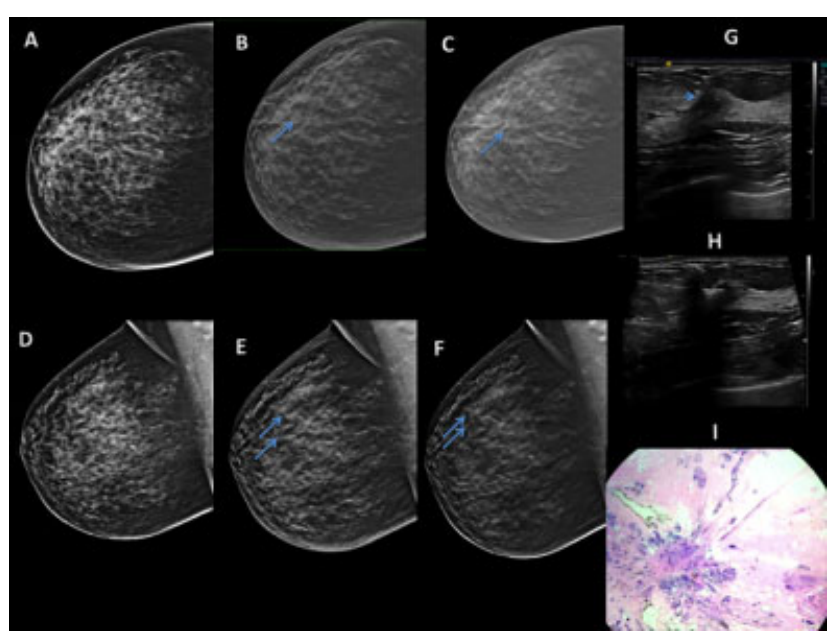

Fig. 11 Screening mammography. Full-field digital mammography (FFDM) (A) of architectural distortion centrally toward the lateral quadrant (arrow) in the right breast which is better appreciated in (B, C) tomosynthesis slice and two-dimensional synthesized mammography (2DSM) images. Note that this is not well identified in the (D) FFDM mediolateral oblique (MLO) view. However, the area is well seen in the (E, F) tomosynthesis slice (double arrows) and 2DSM in MLO projection. Breast Imaging-Reporting and Data System (BI-RADS) 4A. (G) Ultrasound sonography (USG) shows hypoechoic area of architectural distortion (AD) with posterior acoustic shadowing (arrow head); BI-RADS 4B. (H) USG-guided biopsy of the lesion showed (I) radial scar on histopathological examination (HPE).

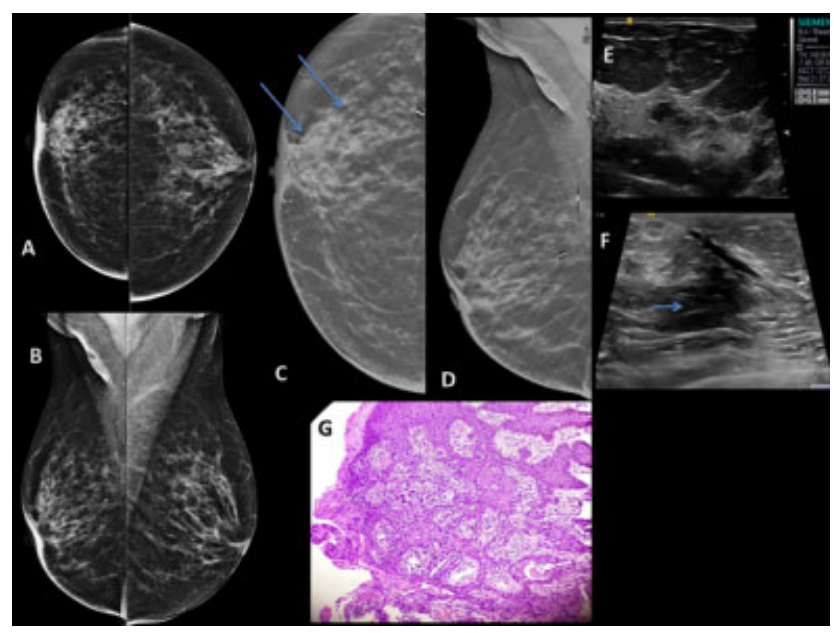

Fig. 12 History of right breast lumpectomy for invasive ductal carcinoma presenting with redness of right nipple. Full-field digital mammography (FFDM) of both breasts (A, B) craniocaudal (CC) and mediolateral oblique (MLO) views show postsurgical status with skin thickening, retraction of nipple with asymmetry, and architectural distortion $(A D)$ in retroareolar region of right breast toward lateral aspect. Right, two-dimensional synthesized mammography (2DSM) (C, D) in CC and MLO views additionally show pleomorphic microcalcifications (double arrows) in the area. (E, F) Ultrasound sonography (USG) reveals dilated duct with irregular hypoechoic area (short arrow). (G) Magnetic resonance microscopy (MRM) was done: histopathological examination (HPE) showed recurrent tumor with pagetoid spread to skin.

The radiating lines in AD that converge to a point are seen in thin slices DBT images and are more conspicuous and are better visualized on the corresponding 2DSM images (-Fig. 9)., ${ }^{2,8}$
These may appear as a primary finding in $12 \%$ of subtle breast cancers noted at DBT. ${ }^{7}$ This finding has a high positive predictive value for cancer in approximately $60 \%$ of the cases. $^{23}$ Durand et al found that cancers seen as ADs on DBT images, were occult in 50\% cases on conventional FFDM (-Fig. 10). ${ }^{22}$

Radial scars (-Fig. 11), sclerosing adenosis, and complex sclerosing lesions are considered pathologically benign but typically manifest as AD which is well identified on 2DSM with a similar appearance to that of malignancy. ${ }^{22}$

Distortion of normal architecture can also be caused by postprocedure scars in cases with a previous history of biopsy, lumpectomy, or mammoplasty surgery. However, suspicious findings are better appreciated on DBT slices with 2DSM with associated postoperative changes that may suggest recurrence of cancer (-Fig. 12).

In cases of BCS, postlumpectomy patients on surveillance, findings of $A D$, and focal asymmetry must be biopsied to rule out malignancy (-Fig. 13A, B).

At times, fat necrosis may undergo fibrosis and appear as AD or focal asymmetry (-Fig. 14).

In women with breast implants, implant-displaced DBT in standard projections of the breast can be performed. The $1 \mathrm{~mm}$ thin tomosynthesis slices help to visualize subtle abnormalities better and 2DSM can reveal AD and fine microcalcifications well (-Fig. 15).

\section{Drawbacks and Limitations}

DBT is essentially a mammographic procedure and the same contraindications and restrictions for candidates whose breasts cannot be compressed adequately or patients who cannot be positioned properly limit the performance of tomosynthesis.

\section{Size}

DBT cannot be performed for very small breasts. The average compressed breast thickness of more than $2 \mathrm{~cm}$ is optimal. This is a problem especially for implant-displaced views for small breasts.

Very large breasts cannot be covered in one acquisition data set. This leads to incomplete visualization of the entire breast in one projection and is a drawback for inadequate coverage for reconstructing C-view images.

\section{Breast Density}

There is limited visualization with extremely dense fibroglandular tissue, which may obscure findings even in thin section tomo-slices. This impacts the quality of the synthesized C-view images also.

\section{Image Resolution}

The main limitation is the resolution of synthesized 2D Cview image is lesser as compared with that for FFDM of the same breast in the respective CC or MLO projection. This also depends on the size and thickness of the compressed breast as well as the exposure factors. 2D FFDM has the highest resolution theoretically with the X-ray tube making 0 -degree angle. The resolution of 2DSM images for our machine is 


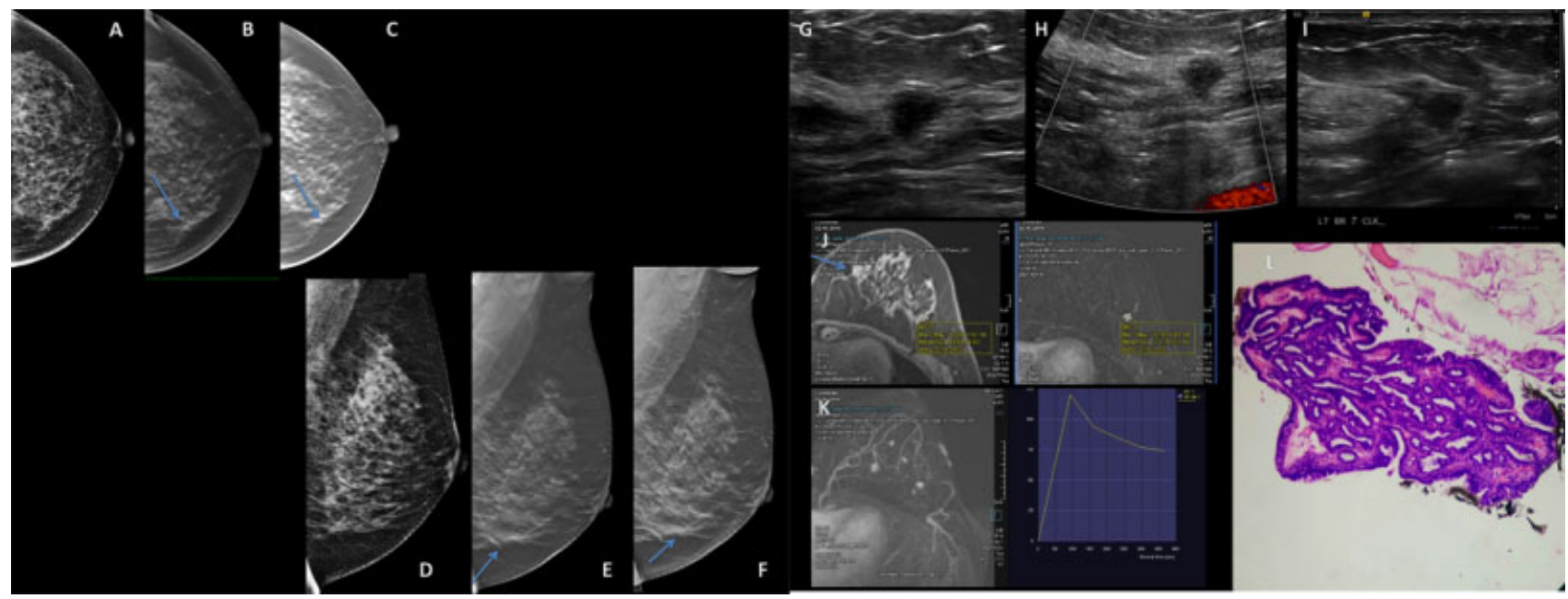

Fig. 13 (A) History of lumpectomy for malignant lesion in right breast for follow-up mammography. Left breast full-field digital mammography (FFDM) in craniocaudal (CC) and mediolateral oblique (MLO) projections (A, D) show focal asymmetry in the left inferomedial quadrant. Associated mild architectural distortion seen well on respective $1 \mathrm{~mm}$ tomosynthesis (B, D) and (C, F) two-dimensional synthesized mammography (2DSM) images (arrows). (G-I) Ultrasound sonography (USG) showed hypoechoic lesion with irregular and microlobulated margins in the left breast at 7 o'clock position; Breast Imaging-Reporting and Data System (BI-RADS) 4B. (J, K) Dynamic contrast-enhanced magnetic resonance imaging (CEMRI) revealed irregular spiculated mass lesion (arrow) in the left breast at the 7 to 8 o'clock position with type 3 curve on kinetics. (L) Histopathological examination (HPE): Benign intraductal papilloma.

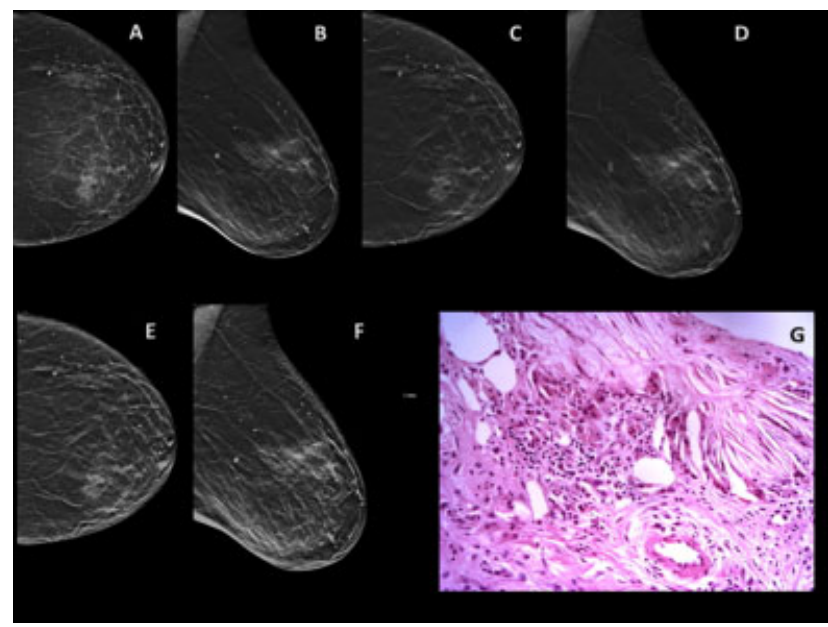

Fig. 14 A 74-year-old lady with history of trauma and tender lump in left breast of 1 month duration. Left breast full-field digital mammography (FFDM) in (A, B) craniocaudal (CC) and mediolateral oblique (MLO) view shows focal asymmetry in superomedial quadrant and coarse benign calcifications of liponecrosis. (C, D) $1 \mathrm{~mm}$ tomosynthesis slice shows architectural distortion (AD). (E, F) Two-dimensional synthesized mammography (2DSM) shows focal lucent areas within this area. Breast Imaging-Reporting and Data System (BIRADS) 4A. (G) Hematoxylin and eosin (H\&E) $200 \times$ fat necrosis.

Inflammatory cells including histiocytes, giant cells, and cholesterol clefts.

approximately 87 to $106 \mu \mathrm{m}$ as compared with the $2 \mathrm{D}$ pixel size of 70 to $76 \mu \mathrm{m}$ for the same breast (-Fig. 16).

\section{Artifacts}

Tomosynthesis and 2DSM images may show significant outof-plane artifacts, skin processing artifacts, terracing artifacts, and calcification or metal artifacts due to attenuation differences (-Fig. 17). ${ }^{24}$ Terracing artifacts are striated lines which are usually seen at the edges of the image perpendic-

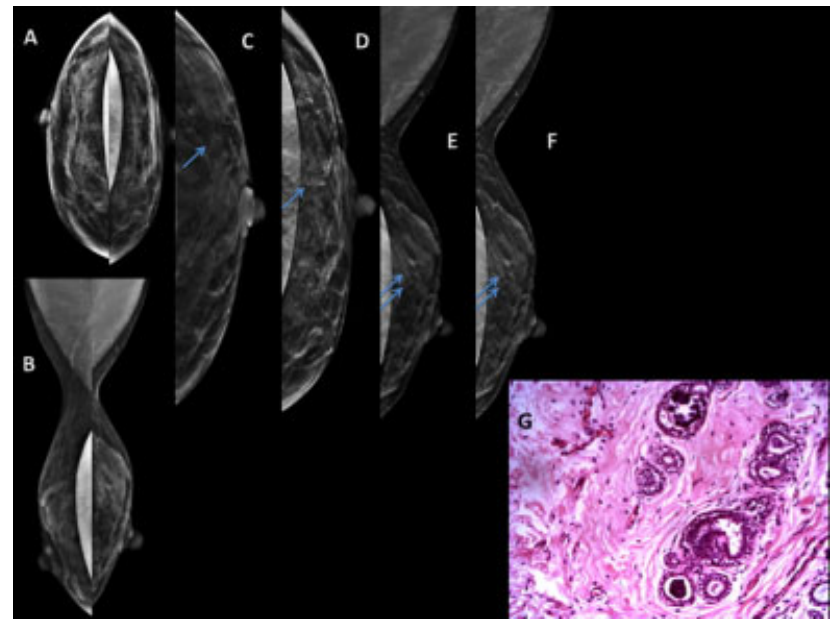

Fig. 15 Screening mammography in a 51-year-old lady. Bilateral breast implants in situ. Implant displaced full-field digital mammography (FFDM) in (A) craniocaudal (CC) and (B) mediolateral (ML) views show microcalcifications in medial aspect of right breast and focal asymmetry with microcalcifications in superolateral quadrant of left breast which is appreciated as architectural distortion (AD) with monomorphic microcalcifications in radial distribution in digital breast tomosynthesis (DBT) $1 \mathrm{~mm}$ tomosynthesis slices and twodimensional synthesized mammography (2DSM) images (C, E) CC (arrow) and in (D, F) ML (double arrows) projections. (G) Hematoxylin and eosin (H\&E) benign ducts with intraluminal microcalcification.

ular to the direction of sweep of the X-ray tube as tissue comes into the field of view on 3D projection. ${ }^{24}$ Some of these can be corrected and others are not so significant, however, need to be recognized by the radiologist.

\section{Patient Radiation}

Increased radiation dose to a patient with DBT is known. The dose for DM is approximately $1.2 \mathrm{mGy}$. Average increase of 


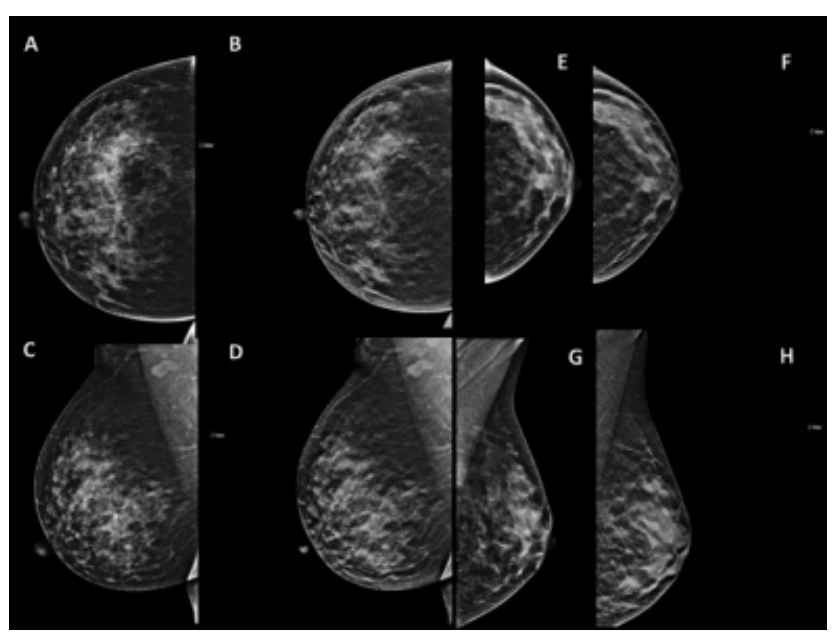

Fig. 16 Full-field digital mammography (FFDM) and two-dimensional synthesized mammography (2DSM) (C-view) are visually comparable in appearance for larger and smaller size breasts. Left breast (A, B), (C, D) and right breast $(\mathrm{E}, \mathrm{F}),(\mathrm{G}, \mathrm{H})$ in craniocaudal $(\mathrm{CC})$ and mediolateral oblique (MLO) images, respectively; the resolution of FFDM $\sim 70-77$ mic and 2DSM (C-view) $\sim 87-106$ mic. Microcalcifications are better appreciated on 2DSM images.

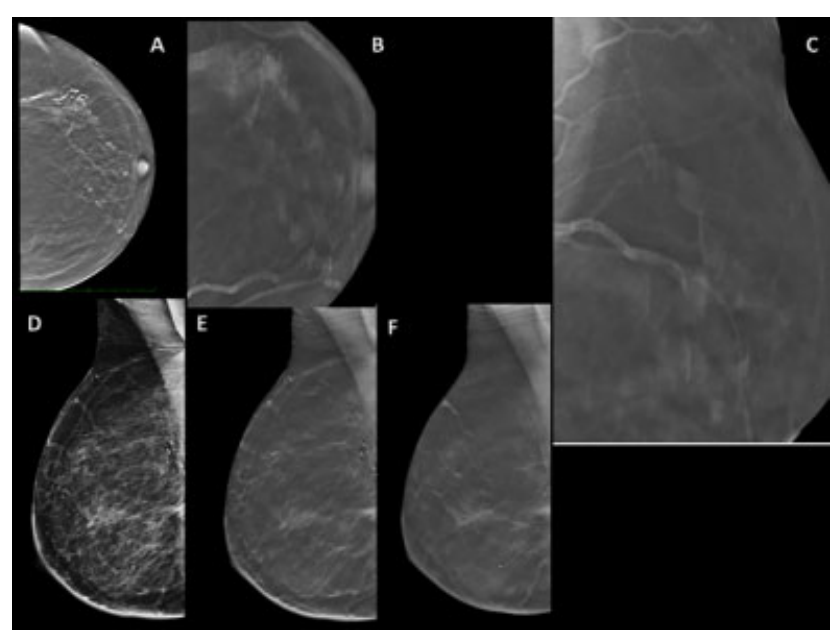

Fig. 17 Tomosynthesis artifacts. Left breast (A, B) craniocaudal (CC) and $(C)$ magnified mediolateral oblique (MLO) view shows terrace artifacts for calcifications. Right breast MLO views (D-F) postlumpectomy status with shadow artifacts at site of surgical clips and skin folds with processing staircase artifacts in right axillary region.

DBT dose compared with FFDM is 38\%, and a range between 0 and $75 \%$ as published in a study by Gennaro et al $^{25}$ compared with the dose at FFDM in combo-mode is up to $2.65 \mathrm{mGy}$. The average patient dose of DBT with 2D C-view dose is $1.46 \mathrm{mGy}$, as shown in a study done on ACR phantom for our system. The additional radiation burden should not deter its use keeping the clinical benefit and advantages in diagnostic value in mind.

\section{Time Factor}

The exposure time is longer in each projection for each breast DBT (up to 4 seconds) and increases up to 10 seconds with add-on standard FFDM exposure with the tube returning to the center after completing the arc along the scan angle.

This adds to patient discomfort with increased compression time for each view and may result in degraded images due to motion blur.

\section{Increased Data}

The files are larger and need more disc space for archiving. More images increase the time for evaluation and interpretation. Many slices in DBT have to be read in conjunction with 2D images, that is, 2DSM and FFDM. It is important that comparison with previous studies is mentioned in the report, with BI-RADS category 0 to 6 , and appropriate recommendations.

\section{Conclusion}

The role of DBT is expanding in screening and diagnostic breast imaging with 2D C-view images being used with tomo-slices. The quality of C-view images is comparable with conventional mammography images, with enhanced visualization of ADs and microcalcifications. Technological advances contribute to improved resolution of 2DSM image resulting in an acceptable diagnostic quality. This may lead to eliminating the need for acquiring both FFDM and DBT data set during tomosynthesis-based screening and thereby reduction of overall radiation dosage. 2D reconstructed images created from DBT with 2DSM has the potential to replace conventional FFDM. ${ }^{26}$

\section{Conflict of Interest}

There are no conflicts of interest.

Financial Support and Sponsorship

None.

\section{Acknowledgments}

None.

\section{References}

1 Tabár L, Yen AM-F, Wu WY-Yet al.Insights from the breast cancer screening trials: how screening affects the natural history of breast cancer and implications for evaluating service screening programs. Breast J 2015;21(01):13-20

2 Hooley RJ, Durand MA, Philpotts LE. Advances in digital breast tomosynthesis. AJR Am J Roentgenol 2017;208(02):256-266

3 Kolb TM, Lichy J, Newhouse JH. Comparison of the performance of screening mammography, physical examination, and breast US and evaluation of factors that influence them: an analysis of 27,825 patient evaluations. Radiology 2002;225(01):165-175

4 Svahn TM, Houssami N, Sechopoulos I, Mattsson S. Review of radiation dose estimates in digital breast tomosynthesis relative to those in two-view full-field digital mammography. Breast 2015;24(02):93-99

5 Skaane P, Bandos AI, Eben EBet al.Two-view digital breast tomosynthesis screening with synthetically reconstructed projection images: comparison with digital breast tomosynthesis with fullfield digital mammographic images. Radiology 2014;271(03): 655-663 
6 Zuley ML, Guo B, Catullo VJ, Chough DM, Kelly AE, Lu AH, et al. Comparison of two-dimensional synthesized mammograms versus original digital mammograms alone and in combination with tomosynthesis images. Radiology 2014;271:664-677

7 Peppard HR, Nicholson BE, Rochman CM. Merchant JK, Mayo RC III, Harvey JA. Digital breast tomosynthesis in the diagnostic setting: indications and clinical applications. Radiographics 2015;35(04):975-990

8 Choi JS, Han BK, Ko EYet al.Comparison between two-dimensional synthetic mammography reconstructed from digital breast tomosynthesis and full-field digital mammography for the detection of T1 breast cancer. Eur Radiol 2016;26(08):2538-2546

9 Sickles EA, D'Orsi CJ, Bassett LWet al. ACR BI-RADS mammography. In: D'Orsi C, Sickles EA, Mendelson EB, Morris EA, eds. ACR BIRADS Atlas Breast Imaging Reporting and Data System 2013. 5th ed. Reston, VA: American College of Radiology; 2013:1-175

10 Zuley ML, Bandos AI, Ganott MAet al.Digital breast tomosynthesis versus supplemental diagnostic mammographic views for evaluation of noncalcified breast lesions. Radiology 2013;266(01): 89-95

11 Dang PA, Freer PE, Humphrey KL, Halpern EF, Rafferty EA. Addition of tomosynthesis to conventional digital mammography: effect on image interpretation time of screening examinations. Radiology 2014;270(01):49-56

12 Freer PE, Wang JL, Rafferty EA. Digital breast tomosynthesis in the analysis of fat-containing lesions. Radiographics 2014;34(02): 343-358

13 Mun HS, Kim HH, Shin HJet al.Assessment of extent of breast cancer: comparison between digital breast tomosynthesis and full-field digital mammography. Clin Radiol 2013;68(12): 1254-1259

14 Luparia A, Mariscotti G, Durando Met al.Accuracy of tumour size assessment in the preoperative staging of breast cancer: comparison of digital mammography, tomosynthesis, ultrasound and MRI. Radiol Med (Torino 2013;118(07):1119-1136

15 Rominger M, Wisgickl C, Timmesfeld N. Breast microcalcifications as type descriptors to stratify risk of malignancy: a systematic review and meta-analysis of 10665 cases with special focus on round/punctate microcalcifications. Röfo Fortschr Geb Röntgenstr Nuklearmed 2012;184(12):1144-1152

16 Rao AA, Feneis J, Lalonde C, Ojeda-Fournier HA pictorial review of changes in the BI-RADS: fifth edition. Radiographics2016; 36:623-639

17 Destounis SV, Arieno AL, Morgan RC. Preliminary clinical experience with digital breast tomosynthesis in the visualization of breast microcalcifications. J Clin Imaging Sci 2013;3:65

18 Spangler ML, Zuley ML, Sumkin JHet al.Detection and classification of calcifications on digital breast tomosynthesis and 2D digital mammography: a comparison. AJR Am J Roentgenol 2011;196(02):320-324

19 Kopans D, Gavenonis S, Halpern E, Moore R. Calcifications in the breast and digital breast tomosynthesis. Breast J 2011;17(06): 638-644

20 Chesebro AL, Winkler NS, Birdwell RL, Giess CS. Developing asymmetries at mammography: a multimodality approach to assessment and management. Radiographics 2016;36(02): 322-334

21 Price ER, Joe BN, Sickles EA. The developing asymmetry: revisiting a perceptual and diagnostic challenge. Radiology 2015;274(03): 642-651

22 Durand MA, Wang S, Hooley RJ, Raghu M, Philpotts LE. Tomosynthesis-detected architectural distortion: management algorithm with radiologic-pathologic correlation. Radiographics 2016;36 (02):311-321

23 Harvey JA, March DEMaking the Diagnosis: A Practical Guide to Breast Imaging. Philadelphia, PA: Elsevier Saunders2013

24 Geiser WR, Einstein SA, Yang W-T. Artifacts in digital breast tomosynthesis. AJR Am J Roentgenol 2018;211(04):926-932

25 Gennaro G, Bernardi D, Houssami N. Radiation dose with digital breast tomosynthesis compared to digital mammography: perview analysis. Eur Radiol 2018;28(02):573-581

26 Choi Y, Woo O-H, Shin H-S, Cho KR, Seo BK, Choi G-Y. Quantitative analysis of radiation dosage and image quality between digital breast tomosynthesis (DBT) with two-dimensional synthetic mammography and full-field digital mammography (FFDM. Clin Imaging 2019;55:12-17 